\title{
A finiteness bound for the EPRL/FK spin foam model
}

\author{
Aleksandar Mikovid* \\ Departamento de Matemática, Universidade Lusófona de Humanidades e Tecnologia, \\ Av. do Campo Grande, 376, 1749-024, Lisboa, Portugal \\ Marko Vojinovid† \\ Grupo de Física Matemática da Universidade de Lisboa, \\ Av. Prof. Gama Pinto, 2, 1649-003 Lisboa, Portugal
}

(Dated: October 18, 2018)

\begin{abstract}
We show that the EPRL/FK spin foam model of quantum gravity has an absolutely convergent partition function if the vertex amplitude is divided by an appropriate power $p$ of the product of dimensions of the vertex spins. This power is independent of the spin foam 2-complex and we find that $p>2$ insures the convergence of the state sum. Determining the convergence of the state sum for the values $0 \leq p \leq 2$ requires the knowledge of the large-spin asymptotics of the vertex amplitude in the cases when some of the vertex spins are large and other are small.
\end{abstract}

PACS numbers: 04.60.Pp

\footnotetext{
*Electronic address: amikovic@ulusofona.pt Member of Grupo de Física Matemática da Universidade de Lisboa, Av. Prof. Gama Pinto, 2, 1649-003 Lisboa, Portugal

${ }^{\dagger}$ Electronic address: vmarko@cii.fc.ul.pt
} 


\section{INTRODUCTION}

Spin foam models are quantum gravity theories where the quantum geometry of spacetime is described by a colored two-complex where the colors are the spins, i.e. the irreducible $S U(2)$ group representations and the corresponding intertwiners. By assigning appropriate weights for the simplexes of the 2-complex and by summing over the spins and the intertwiners, one obtains a state sum that can be interpreted as the transition amplitude for the boundary quantum geometries, which are described by spin networks [1]. A spin foam state sum can be considered as a path integral for general relativity.

The most advanced spin foam model constructed so far is the EPRL/FK model, introduced in [2, 3]. The finiteness, as well as the semiclassical properties of a spin foam model, depend on the large-spin asymptotics of the vertex amplitude. This asymptotics was studied in [4 6] for the EPRL/FK case. The study of the finiteness of the model was started in [7], where only two simple spin foam amplitudes were studied (equivalent to loop Feynman diagrams with 2 and 5 vertices) in the Euclidean case. It was concluded that the degree of divergence of these two spin foam transition amplitudes depends on a choice of the normalization of the vertex amplitude. This normalization is a power of the product of the dimensions of the spins and the intertwiners which label the faces and the edges of the 4-simplex dual to a spin-foam vertex.

One can exploit this freedom in the definition of the EPRL/FK vertex amplitude in order to achieve the finiteness of the model. Namely, an EPRL/FK vertex amplitude can be introduced such that it is the original one divided by a positive power $p$ of the product of dimensions of the vertex spins $\Delta_{v}$. This new amplitude will give the state sum with better convergence properties, and one can try to find a range of $p$ for which the state sum is convergent. In this paper we will show that there are such values of $p$ which are independent from the spin-foam 2-complex.

Note that an equivalent approach was used in the case of the Barrett-Crane spin foam model, where the finiteness was achieved by introducing an appropriate edge amplitude [8, 9]. This is an equivalent approach to our approach because a state sum with a dual edge amplitude $A_{3}(j)=\left(\operatorname{dim} j_{1} \ldots \operatorname{dim} j_{4}\right)^{q}$ and a vertex amplitude $A_{4}(j)$ is the same as the state sum with $\tilde{A}_{3}(j)=1$ and $\tilde{A}_{4}(j)=\left(\Delta_{v}\right)^{p}(j) A_{4}(j)$, where $p$ is an appropriate power.

Our paper is organized such that in section \we describe the EPRL/FK spin foam model 
and discuss the large-spin asymptotic properties of the vertex amplitude. In section [II we show that the vertex amplitude divided by the product of the dimensions of the vertex spins is a bounded function of the spins. In section [V] we introduce a rescaled EPRL/FK vertex amplitude, which is the original amplitude divided by the product of the dimensions of the vertex spins raised to a power $p$. We prove that the corresponding state sum is absolutely convergent for $p>2$ by using the amplitude estimate from section [II], In section $\nabla$ we discuss our results and present conclusions.

\section{THE VERTEX AMPLITUDE}

The EPRL/FK spin foam model state sum is given by

$$
Z(T)=\sum_{j, \iota} \prod_{f \in T^{*}} A_{2}\left(j_{f}\right) \prod_{v \in T^{*}} W\left(j_{f(v)}, \iota_{e}(v)\right)
$$

where $T$ is a triangulation of the spacetime manifold, $T^{*}$ is the dual simplicial complex, while $e, f$ and $v$ denote the edges, the faces and the vertices of $T^{*}$, respectively. The sum in (1) is over all possible assignements of $S U(2)$ spins $j_{f}$ to the faces of $T^{*}$ (triangles of $T$ ) and over the corresponding intertwiner assignemets $\iota_{e}$ to the edges of $T^{*}$ (tetrahedrons of T). $A_{2}$ is the face amplitude, and it can be fixed to be

$$
A_{2}(j)=\operatorname{dim} j=2 j+1
$$

by using the consistent glueing reguirements for the transition amplitudes between threedimensional boundaries, see [12].

The vertex amplitude $W$ can be written as

$$
W\left(j_{f}, \iota_{e}\right)=\sum_{k_{e} \geq 0} \int_{0}^{+\infty} d \rho_{e}\left(k_{e}^{2}+\rho_{e}^{2}\right)\left(\bigotimes_{e} f_{k_{e} \rho_{e}}^{\iota_{e}}\left(j_{f}\right)\right)\{15 j\}_{S L(2, \mathbb{C})}\left(\left(2 j_{f}, 2 \gamma j_{f}\right) ;\left(k_{e}, \rho_{e}\right)\right),
$$

where the $15 j$ symbol is for the unitary representations $(k, \rho)$ of the $S L(2, C)$ group, the universal covering group of the Lorentz group. The $f_{k_{e} \rho_{e}}^{\iota_{e}}$ are the fusion coefficients, defined in detail in [2, 3, 10].

Instead of using the spin-intertwiner basis, one can rewrite (1) in the coherent state basis, introduced in [11]. In this basis, the state sum is given by

$$
Z(T)=\sum_{j} \int \prod_{e, f} d^{2} \vec{n}_{e f} \prod_{f \in T^{*}} \operatorname{dim} j_{f} \prod_{v \in T^{*}} W\left(j_{f(v)}, \vec{n}_{e(v) f(v)}\right)
$$


The $\vec{n}_{e f}$ is a unit three-dimensional vector associated to the triangle dual to a face $f$ of the tetrahedron dual to an edge $e$ which belongs to $f$ (see [11] for details). For a geometric tetrahedron, the four vectors $\vec{n}$ can be identified with the unit normal vectors for the triangles. Note that the domain of integration for each such vector is a 2-sphere.

The key property of $W(j, \vec{n})$ amplitude, which was used to find the large-spin asymptotics, is that it can be written as an integral over the manifold $S L(2, \mathbb{C})^{4} \times\left(\mathbb{C P}^{1}\right)^{10}$, see [6]. More precisely,

$$
W(j, \vec{n})=\text { const. } \prod_{k=1}^{10} \operatorname{dim} j_{k} \int_{S L(2, \mathbb{C})^{5}} \prod_{a=1}^{5} d g_{a} \delta\left(g_{5}\right) \int_{\left(\mathbb{C P}^{1}\right)^{10}} \prod_{k=1}^{10} d z_{k} \Omega(g, z) e^{S(j, \vec{n}, g, z)}
$$

where $\Omega$ is a slowly changing function and

$$
S(j, \vec{n}, g, z)=\sum_{k=1}^{10} j_{k} \log w_{k}(\vec{n}, g, z)=\sum_{k=1}^{10} j_{k}\left(\ln \left|w_{k}(\vec{n}, g, z)\right|+i \theta_{k}(\vec{n}, g, z)\right) .
$$

The functions $w_{k}$ are complex-valued, so that $\theta_{k}=\arg w_{k}+2 \pi m_{k}$, where $m_{k}$ are integers which have to be chosen such that $\log w_{k}$ belong to the same branch of the logarithm.

Since $\left|w_{k}\right| \leq 1$, it follows that $R e S \leq 0$ and it can be shown that the large-spin asymptotics is given by

$$
W(\lambda j, \vec{n}) \approx \frac{\text { const }}{\lambda^{12}} \sum_{x^{*}} \frac{\Omega\left(x^{*}\right) e^{i \lambda \sum_{k} j_{k} \theta_{k}\left(\vec{n}, x^{*}\right)}}{\sqrt{\operatorname{det}\left(-H\left(j, \vec{n}, x^{*}\right)\right)}},
$$

for $\lambda \rightarrow+\infty$, where the sum is over the critical points $x^{*}=\left(g^{*}, z^{*}\right)$ satisfying

$$
\operatorname{Re} S\left(j, \vec{n}, g^{*}, z^{*}\right)=0,\left.\quad \frac{\partial S}{\partial g_{a}}\right|_{x^{*}}=0,\left.\quad \frac{\partial S}{\partial z_{k}}\right|_{x^{*}}=0
$$

and $H(x)$ is the Hessian for the function $S(x)$. There are finitely many critical points, and it can be shown that the conditions (6) require that $j_{k}$ are proportional to the areas of triangles for a geometric 4-simplex, while $\vec{n}$ have to be the normal vectors for the triangles in a tetrahedron of a geometric 4-simplex and $g^{*}$ have to be the corresponding holonomies. A geometric 4-simplex has a consistent assigment of the edge-lengths, and it can be shown that $\theta_{k}\left(\vec{n}, x^{*}\right)$ is proportional to the dehidral angle for a triangle in a geometric 4-simplex, so that

$$
S_{R}^{(v)}=\sum_{k=1}^{10} j_{k} \theta_{k}\left(\vec{n}, x^{*}\right)
$$

corresponds to the Regge action for a 4-simplex. 
The Hessian $H(j, \vec{n}, x)$ is a $44 \times 44$ matrix, and

$$
H_{\alpha \beta}\left(j, \vec{n}, x^{*}\right)=\sum_{k=1}^{10} j_{k} H_{\alpha \beta}^{(k)}\left(\vec{n}, x^{*}\right),
$$

since $S$ is a linear function of $j$. Consequently

$$
\operatorname{det}(-H)=\sum_{m_{1}+\cdots+m_{10}=44}\left(j_{1}\right)^{m_{1}} \cdots\left(j_{10}\right)^{m_{10}} D_{m_{1} \ldots m_{10}}\left(\vec{n}, x^{*}\right)
$$

is a homogeneous polinomial of degree 44 in $j_{k}$ variables. One also has that $R e(-H)$ is a positive definite matrix.

\section{A BOUND FOR THE VERTEX AMPLITUDE}

We will now find a bound for the vertex amplitude by using the asymptotic formula (5) and its generalization for the case when some of the vertex spins are large and other are small. Since $\lambda S(j, \vec{n}, x)=S(\lambda j, \vec{n}, x)$ and

$$
\lambda^{44} \operatorname{det}\left(-H\left(j, \vec{n}, x^{*}\right)\right)=\operatorname{det}\left(-H\left(\lambda j, \vec{n}, x^{*}\right)\right)
$$

then the formula (5) can be rewritten as

$$
W(j, \vec{n}) \approx \mathrm{const} \prod_{k=1}^{10} \operatorname{dim} j_{k} \sum_{x^{*}} \frac{\Omega\left(x^{*}\right) e^{i \sum_{k} j_{k} \theta_{k}\left(\vec{n}, x^{*}\right)}}{\sqrt{\operatorname{det}\left(-H\left(j, \vec{n}, x^{*}\right)\right)}}
$$

when $j=\left(j_{1}, \ldots, j_{10}\right) \rightarrow(+\infty, \ldots,+\infty) \equiv(+\infty)^{10}$, because $\prod_{k=1}^{10} \operatorname{dim} j_{k}$ scales as $\lambda^{10}$ for $\lambda$ large. Therefore

$$
\lim _{j \rightarrow(+\infty)^{10}} W(j, n)=\mathrm{const} \lim _{j \rightarrow(+\infty)^{10}} \prod_{k=1}^{10} \operatorname{dim} j_{k} \sum_{x^{*}} \frac{\Omega\left(x^{*}\right) e^{i \sum_{k} j_{k} \theta_{k}\left(\vec{n}, x^{*}\right)}}{\sqrt{\operatorname{det}\left(-H\left(j, \vec{n}, x^{*}\right)\right)}} .
$$

Note that

$$
\left|\sum_{x^{*}} \frac{\Omega\left(x^{*}\right) e^{i \sum_{k} j_{k} \theta_{k}\left(\vec{n}, x^{*}\right)}}{\sqrt{\operatorname{det}\left(-H\left(j, \vec{n}, x^{*}\right)\right)}}\right| \leq \sum_{x^{*}} \frac{\left|\Omega\left(x^{*}\right)\right|}{\sqrt{\mid \operatorname{det}\left(-H\left(j, \vec{n}, g^{*}\right)\right)}},
$$

and

$$
\lim _{j \rightarrow(+\infty)^{10}} \frac{\prod_{k=1}^{10} \operatorname{dim} j_{k}}{\sqrt{\left|\operatorname{det}\left(-H\left(j, \vec{n}, x^{*}\right)\right)\right|}}=0,
$$

due to (8). The equations (9),(10) and (11) imply

$$
\lim _{j \rightarrow(+\infty)^{10}} W(j, \vec{n})=0
$$


The equation (12) is equivalent to

$$
\forall \epsilon>0, \exists \delta>0 \quad \text { such that } \quad j_{1}>\delta, \cdots, j_{10}>\delta \Rightarrow|W(j, \vec{n})|<\epsilon
$$

This implies that $W$ is a bounded function in the region

$$
D_{10}=\left\{j \mid j_{1}>\delta, \cdots, j_{10}>\delta\right\}
$$

If we denote with $D_{m}$ the region where $m<10$ spins are greater than $\delta$ and the rest are smaller or equal than $\delta$, then

$$
\mathbb{R}_{+}^{10} \backslash D_{10}=\bigcup_{m=0}^{9} D_{m}
$$

Since the regions $D_{m}$ are not compact for $m>0$, we do not know whether $W$ is bounded in these regions. In order to determine this we need to know the asymptotics of $W$ for the cases when some of the spins are large and other are small. This asymptotics can be obtained by using the same method as in the case when all the vertex spins are large.

Let $m$ be the number of large spins ( $m \geq 3$ due to the triangle inequalities for the vertex spins) and let $j^{\prime}=\left(j_{1}, \ldots, j_{m}\right)$. Then

$$
S\left(\lambda j^{\prime}, j^{\prime \prime}, n, x\right)=\sum_{k=1}^{m} \lambda j_{k}^{\prime}\left(\ln \left|w_{k}\right|+i \theta_{k}\right)+\sum_{k=m+1}^{10} j_{k}^{\prime \prime}\left(\ln \left|w_{k}\right|+i \theta_{k}\right)=\lambda S_{m}\left(j^{\prime}, n, x\right)+O(1) .
$$

Therefore the asymptotic properties of $W\left(j^{\prime}, j^{\prime \prime}, n\right)$ will be determined by the critical points of $S_{m}\left(j^{\prime}, n, x\right)$. Consequently

$$
W\left(\lambda j^{\prime}, j^{\prime \prime}, \vec{n}\right) \approx \frac{\mathrm{const}}{\lambda^{r / 2-m}} \sum_{x^{*}} \frac{\Omega\left(x^{*}\right) e^{i \lambda \sum_{k=1}^{m} j_{k}^{\prime} \theta_{k}\left(\vec{n}, x^{*}\right)}}{\sqrt{\operatorname{det}\left(-\tilde{H}_{m}\left(j^{\prime}, \vec{n}, x^{*}\right)\right)}}
$$

where $r$ is the rank of the Hessian matrix $H_{m}$ for $S_{m}$ at a critical point $x^{*}(1 \leq r \leq 44)$ and $\tilde{H}_{m}$ is the reduced Hessian matrix. $\tilde{H}_{m}$ is the restriction of the Hessian $H_{m}$ to the orthogonal complement of $\mathrm{Ker} H_{m}$ and $\tilde{H}_{m}$ has to be used if $r<44$.

The asymptotics (13) implies that the function $W\left(j^{\prime}, j^{\prime \prime}, \vec{n}\right)$ will vanish for large $j^{\prime}$ if $r / 2-m>0$. If this was true for all $m$ we could say that $W(j)$ is a bounded function in $\mathbb{R}_{+}^{10}$. However, calculating the values for $r$ is not easy. Instead, we are going to estimate $\left|W\left(j^{\prime}, j^{\prime \prime}, \vec{n}\right)\right|$. Note that (13) is equivalent to

$$
W\left(j^{\prime}, j^{\prime \prime}, \vec{n}\right) \approx \mathrm{const} \prod_{k=1}^{m} \operatorname{dim} j_{k}^{\prime} \sum_{x^{*}} \frac{\Omega\left(x^{*}\right) e^{i \lambda \sum_{k=1}^{m} j_{k}^{\prime} \theta_{k}\left(\vec{n}, x^{*}\right)}}{\sqrt{\operatorname{det}\left(-\tilde{H}_{m}\left(j^{\prime}, \vec{n}, x^{*}\right)\right)}}
$$


for $j^{\prime} \rightarrow(+\infty)^{m}$, since $S_{m}$ and $\tilde{H}_{m}$ are linear functions of the spins $j^{\prime}$ and $\operatorname{det}\left(-\tilde{H}_{m}\right)$ scales as $\lambda^{r}$, while $\prod_{k=1}^{m} \operatorname{dim} j_{k}$ scales as $\lambda^{m}$ when $j^{\prime} \rightarrow \lambda j^{\prime}$ and $\lambda$ is large. Hence

$$
\frac{W\left(j^{\prime}, j^{\prime \prime}, \vec{n}\right)}{\prod_{k=1}^{m} \operatorname{dim} j_{k}^{\prime}} \approx \text { const } \sum_{x^{*}} \frac{\Omega\left(x^{*}\right) e^{i \lambda \sum_{k=1}^{m} j_{k}^{\prime} \theta_{k}\left(\vec{n}, x^{*}\right)}}{\sqrt{\operatorname{det}\left(-\tilde{H}_{m}\left(j^{\prime}, \vec{n}, x^{*}\right)\right)}}
$$

for $j^{\prime} \rightarrow(+\infty)^{m}$.

From here it follows that for every $m \geq 3$

$$
\lim _{j \rightarrow(+\infty)^{m}} \frac{W\left(j^{\prime}, j^{\prime \prime}, \vec{n}\right)}{\prod_{k=1}^{m} \operatorname{dim} j_{k}^{\prime}}=0
$$

since $r(m) \geq 1$. Given that $W=0$ in $D_{1}$ and $D_{2}$, it follows that $W(j, \vec{n}) / \prod_{k=1}^{10} \operatorname{dim} j_{k}$ is a bounded function in $\mathbb{R}_{+}^{10}$. Therefore exists $C>0$ such that

$$
\frac{|W(j, \vec{n})|}{\prod_{k=1}^{10} \operatorname{dim} j_{k}} \leq C
$$

This bound can be rewritten as

$$
|W(j, \vec{n})| \leq C \prod_{k=1}^{10} \operatorname{dim} j_{k}
$$

which is convenient for investigating the absolute convergence of the state sum.

\section{FINITENESS}

We showed in the previous section that the vertex amplitude divided by the product of the dimensions of the vertex spins is a bounded function of spins. This result suggests to introduce a rescaled vertex amplitude $W_{p}$ as

$$
W_{p}\left(j_{f}, \vec{n}_{e f}\right)=\frac{W\left(j_{f}, \vec{n}_{e f}\right)}{\prod_{f=1}^{10}\left(\operatorname{dim} j_{f}\right)^{p}},
$$

where $p \geq 0$, in order to improve the convergence of the state sum.

Given a triangulation $T$ of a compact four-manifold $M$, we will consider the following state sum

$$
Z_{p}=\sum_{j_{f}} \int \prod_{e, f} d^{2} \vec{n}_{e f} \prod_{f \in T^{*}} \operatorname{dim} j_{f} \prod_{v \in T^{*}} W_{p}\left(j_{f(v)}, \vec{n}_{e(v) f(v)}\right) .
$$

It is sufficient to consider $T$ without a boundary, since if $Z(T)$ is finite, then $Z(\Gamma, T)$ will be finite due to gluing properties, where $\Gamma$ is the boundary spin network. 
The convergence of $Z_{p}$ will be determined by the large-spin asymptotics of the vertex amplitude $W$ and the values of $p$. Since the asymptotics of $W$ is not known completely, we will use the estimate (15) in order to find the values of $p$ which make the state sum $Z_{p}$ convergent.

Since

$$
\left|Z_{p}\right| \leq \sum_{j_{f}} \int \prod_{e, f} d^{2} \vec{n}_{e f} \prod_{f \in T^{*}} \operatorname{dim} j_{f} \prod_{v \in T^{*}} \frac{\left|W\left(j_{f(v)}, \vec{n}_{e(v) f(v)}\right)\right|}{\prod_{f \in v}\left(\operatorname{dim} j_{f(v)}\right)^{p}},
$$

and by using (15) we obtain

$$
\left|Z_{p}\right| \leq C^{V} \sum_{j_{f}} \int \prod_{e, f} d^{2} \vec{n}_{e f} \prod_{f \in T^{*}} \operatorname{dim} j_{f} \prod_{v \in T^{*}} \frac{1}{\prod_{f \in v}\left(\operatorname{dim} j_{f(v)}\right)^{p-1}},
$$

where $V$ is the total number of vertices in the triangulation $T$. At this point the integrand does not depend anymore on $\vec{n}_{e f}$, so the appropriate integration over $4 E 2$-spheres can be performed. Here $E$ is the total number of edges in $\sigma$, and it is multiplied by 4 since every edge is a boundary for exactly four faces. After the integration we obtain

$$
\left|Z_{p}\right| \leq C^{V}(4 \pi)^{4 E} \sum_{j_{f}} \prod_{f \in T^{*}} \operatorname{dim} j_{f} \prod_{v \in T^{*}} \frac{1}{\prod_{f \in v}\left(\operatorname{dim} j_{f(v)}\right)^{p-1}} .
$$

The sum over the spins in (19) can be rewritten as a product of single-spin sums. Let $N_{f}$ be the number of vertices bounding a given face $f$. Each vertex contributes with a factor $\left(\operatorname{dim} j_{f}\right)^{-p+1}$, so the total contribution for each face $f$ is $\left(\operatorname{dim} j_{f}\right)^{1-(p-1) N_{f}}$. Thus we can rewrite (19) as

$$
\left|Z_{p}\right| \leq C^{V}(4 \pi)^{4 E} \prod_{f \in T^{*}} \sum_{j_{f} \in \frac{\mathbb{N}_{0}}{2}}\left(\operatorname{dim} j_{f}\right)^{1-(p-1) N_{f}} .
$$

The sum in (20) will be convergent if

$$
1-(p-1) N_{f}<-1
$$

or

$$
p-1>\frac{2}{N_{f}}
$$

for every $N_{f}$. Since $N_{f} \geq 2$ for every face $f$, a sufficient condition for $p$ is

$$
p>2 .
$$

Therefore $Z_{p}$ is absolutely convergent for $p>2$, which means that it is convergent for $p>2$. As far as the convergence of $Z_{p}$ for $p \leq 2$ cases is concerned, one has to calculate the 
ranks of the Hessians $H_{m}$ and use the following inequalities

$$
\left|\operatorname{det}\left(-\tilde{H}_{m}\right)\right| \geq C_{m}\left(\prod_{k=1}^{m} \operatorname{dim} j_{k}\right)^{r / m}
$$

when possible. We expect that the inequalities (23) will hold for all $m$, since $\operatorname{det}\left(-\tilde{H}_{m}\right)$ is a homogeneous polinomial of the spins of the degree $r$ and $\operatorname{Re}\left(-\tilde{H}_{m}\right)$ is a positive definite matrix. Then

$$
|W(j, \vec{n})| \leq C_{q}\left(\prod_{k=1}^{10} \operatorname{dim} j_{k}\right)^{1-q},
$$

for any $j$, where $q=\min \{r / 2 m \mid m=3, \ldots, 10\}$. Since $q>0$, the new bound (24) will be an improvment of the bound (15) and consequently $Z_{p}$ will be absolutely convergent for

$$
p>2-q
$$

Given that $r=44$ for $m=10$, this implies that $q \geq 1 / 18(r=1$ and $m=9$ case $)$ and therefore $p>35 / 18$.

\section{CONCLUSIONS}

We proved that the deformed partition function $Z_{p}$ for the EPRL-FK spin foam model is convergent for $p>2$. We expect that the bound for $p$ can be lowered below 2 , since the inequalities (23) are likely to be true. In this way one can obtain that $p>35 / 18$ without calculating the matrices $H_{m}$.

In order to find the exact value for $q$, the ranks $r$ of the Hessians $H_{m}$ have to be calculated. If it turns out that $q>2$, then the formula (25) will give that the $p=0$ case is convergent. However, if it turns out that $q \leq 2$, then the convergence of the $p=0$ case has to be checked by some other method.

If the $p=0$ state sum is finite, our construction provides an infinite number of new models with better convergence properties. In any case, one has to decide which choices for $p$ are physical. This can be done by analyzing the semiclassical limit of the corresponding EPRL/FK model. As shown in [13, 14], the parameter $p$ appears in the first-order quantum correction to the classical Einstein-Hilbert term. It is therefore an experimental question to determine the value of $p$, provided that quantum gravity is described by an EPRL/FK spin foam model. 
Given that a $p$-deformed spin foam model is finite for $p>2$ and any choice of the triangulation $T$, one can construct a quantum field theory whose Feynman diagrams are in one-to-one correspondence with the transition amplitudes for all triangulations $T$, see [15, 16]. Since all those amplitudes are finite by construction, the corresponding quantum field theory will be perturbatively finite. For such a theory, no regularization scheme is necessary and there is no necessity for a perturbative renormalization procedure.

As the final remark, note that

$$
Z(T)=\sum_{T^{\prime} \subset T} Z^{\prime}\left(T^{\prime}\right)
$$

where $T^{\prime}$ is a sub-complex of $T$ obtained by removing one or more faces from $T$ and $Z^{\prime}$ is the state sum where the zero spins are absent. The state sums $Z^{\prime}$ are considered more physical, because their spin foams correspond to simplicial complex geometries where all the triangles have a non-zero area. The relation (26) was used in [17] to define the sum over the spin foams, since if one chooses a very large $\sigma$, then (26) implies that $Z(\sigma)$ is the result of a sum of the physical transition amplitudes for various spin foams. Since $Z(\sigma)$ can be made finite for EPRL/FK model if one modifies the vertex amplitude as (16), one arrives at a concrete realization of the idea of summing over spin foams.

\section{Acknowledgments}

We would like to thank John Barrett for discussions. AM was partially supported by the FCT grants PTDC/MAT/69635/2006 and PTDC/MAT/099880/2008. MV was supported by grant SFRH/BPD/46376/2008 and partially by PTDC/MAT/099880/2008.

[1] C. Rovelli, Quantum Gravity, Cambridge University Press, Cambridge (2004).

[2] J. Engle, E. Livine, R. Pereira and C. Rovelli, Nucl. Phys. B799 136 (2008), arXiv:0711.0146.

[3] L. Freidel and K. Krasnov, Class. Quant. Grav. 25125018 (2008), arXiv:0708.1595.

[4] F. Conrady and L. Freidel, Phys. Rev. D 78104023 (2008), arXiv:0809.2280.

[5] J. Barrett, R. Dowdall, W. Fairbairn, H. Gomes and F. Hellmann, J. Math. Phys. 50112504 (2009), arXiv:0902.1170. 
[6] J. Barrett, R. Dowdall, W. Fairbairn, F. Hellmann and R. Pereira, Class. Quant. Grav. 27 (2010) 165009, arXiv:0907.2440

[7] C. Perini, C. Rovelli and S. Speziale, Phys. Lett. B 68278 (2009), arXiv:0810.1714.

[8] L. Crane, A. Perez and C. Rovelli, Phys. Rev. Lett. 87181301 (2001), arXiv:gr-qc/0104057.

[9] J. Baez, J. Christensen, T. Halford and D. Tsang, Class. Quant. Grav. 194627 (2002), arXiv:gr-qc/0202017.

[10] F. Conrady and L. Freidel, Class. Quant. Grav. 25245010 (2008), arXiv:0806.4640.

[11] E. R. Livine, S. Speziale, Phys. Rev. D 76084028 (2007), arXiv:0705.0674.

[12] E. Bianchi, D. Regoli and C. Rovelli, arXiv:1005.0764.

[13] A. Miković and M. Vojinović, Class. Quant. Grav. 28225004 (2011), arXiv:1104.1384.

[14] A. Miković and M. Vojinović, J. Phys.: Conf. Ser., 360012049 (2012), arXiv:1110.6114.

[15] A. Miković, Class. Quant. Grav. 182827 (2001).

[16] L. Friedel, Int. J. Theor. Phys. 441769 (2005).

[17] C. Rovelli and M. Smerlak, Class. Quant. Grav. 29055004 (2012), arXiv:1010.5437. 